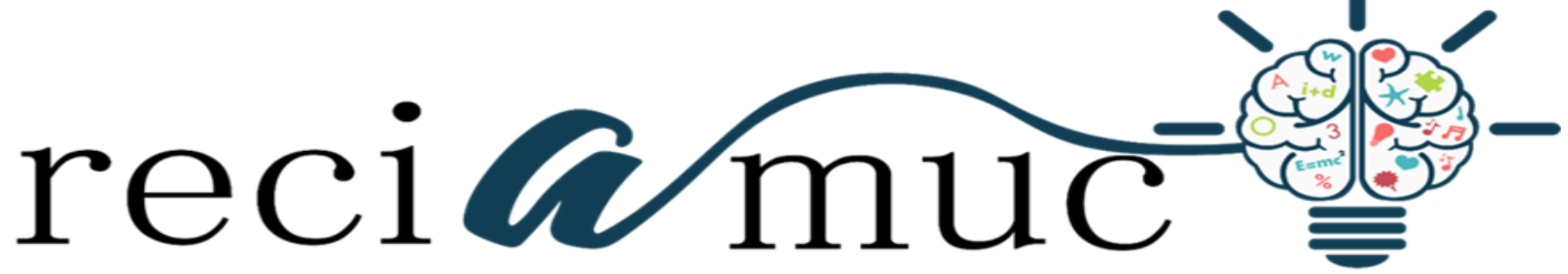

Revista científica de investígación actualización del mundo de las ciencias

Olga Mariana Villavicencio Chica ${ }^{\text {a }}$; Flor María Buri Satama ${ }^{\text {b; }}$ Guido Yunior

García Loor c; Nancy Monserrate Macías Palacios d;

Paulo Gregorio Flores Tacle ${ }^{\mathrm{e}}$; Anthony James Chávez Salazar ${ }^{\mathrm{f}}$

Procedimientos adecuados para pacientes con isquemia e infarto miocardio al ingreso en la UCI

Appropriate procedures for patients with ischemia and myocardial infarction upon admission to the ICU

Revista Científica de Investigación actualización del mundo de las Ciencias. Vol. 3 núm., 3, julio, ISSN: 2588-0748, 2018, pp. 1444-1466

DOI: $\underline{10.26820 / \text { reciamuc/3.(3).julio.2019.1444-1466 }}$

URL: http://reciamuc.com/index.php/RECIAMUC/article/view/402

Código UNESCO: 3205 Medicina Interna

Tipo de Investigación: Artículo de Investigación

(C) RECIAMUC; Editorial Saberes del Conocimiento, 2019

Recibido: 28/04/2019

Aceptado: 19/05/2019

Publicado: 01/07/2019

Correspondencia: olga.villavicencio.ch@gmail.com

a. Médico Cirujano; Médico Residente de Terapia Intensiva Hospital Dr. Gustavo Domínguez; Santo Domingo - Ecuador; olga.villavicencio.ch@gmail.com

b. Médico; Hospital Básico Catacocha - Emergencia; Médico Ecu 911; Loja - Ecuador. marifloriss@ hotmail.com

c. Médico Cirujano; Residente de Emergencia - Hospital Verdi Cevallos Balda; Portoviejo - Ecuador; guido_g191@ @otmail.com

d. Médico Cirujano; Hospital General IESS; Manta - Ecuador; dranancymacias@ yahoo.es

e. Médico - Especialista en Cardiología; Médico tratante del Servicio de Cardiología Hospital IESS Sur; Quito - Ecuador; pgft85@yahoo.es;

f. Doctor en Medicina; Médico Residente de Anestesia Hospital Marcos Vinicio Iza; Lago Agrio - Ecuador; anthonyjames.med@gmail.com 


\section{Procedimientos adecuados para pacientes con isquemia e infarto miocardio al ingreso en la UCI}

Vol. 3, núm. 3., (2019) Olga Mariana Villavicencio Chica; Flor María Buri Satama; Guido Yunior García Loor; Nancy Monserrate Macías Palacios; Paulo Gregorio Flores Tacle; Anthony James Chávez Salazar

\section{RESUMEN}

Algunas enfermedades cardiovasculares (ECV), suelen ser fenómenos agudos originados por obstrucciones que imposibilitan que la sangre fluya hacia el cerebro o el corazón, tal es el caso de los ataques cardiacos, pues los origina un coágulo que bloquea la arteria coronaria que llevan sangre y oxígeno al corazón. A raíz de este bloqueo sanguíneo surge falta de oxígeno, por lo que las células cardíacas mueren y así se genera el daño al músculo cardiaco, siendo todo este proceso conocido como isquemia. Dependiendo del grado de obstrucción de la arteria (total o parcial) será el daño o consecuencia para el organismo, es decir, en aquellos casos de obstrucción grave de la arteria coronaria y de forma repentina, podría causar un ataque cardíaco o infarto al miocardio. El interés por desarrollar la presente investigación que, teniendo como objetivo principal la exposición de los aspectos fundamentales asociados a los procedimientos adecuados en UCI para tratar pacientes con isquemia e infarto al miocardio, y en base a ello, se ha escogido adelantar una investigación bajo un diseño bibliográfico, con una metodología de revisión. En las conclusiones se destacó la exigencia que, hoy más que antes, se demanda para el conocimiento, prevención y tratamientos, no solo del paciente crítico, sino especialmente de las posibles complicaciones asociadas con la enfermedad cardiovascular que por cierto pueden llegar a ser potencialmente graves, y causar la muerte, no obstante, algunas de estas complicaciones pueden prevenirse de manera sencilla, como por ejemplo, con el manejo y la constante actualización de las guías prácticas clínicas elaboradas con base a evidencias comprobadas en UCI. No obstante, el diagnóstico y tratamiento precoz de la isquemia e infarto de miocardio son la clave para evitar las consecuencias irreversibles de la enfermedad, así como el seguimiento de los procedimientos adecuados en la UCI representan la mejor alternativa para la prevención de complicaciones y la recuperación total del paciente.

Palabras Claves: Cardiovasculares; Obstrucciones; Arteria; Células; Paciente. 


\title{
Procedimientos adecuados para pacientes con isquemia e infarto miocardio al ingreso en la UCI
}

Vol. 3, núm. 3., (2019)

Olga Mariana Villavicencio Chica; Flor María Buri Satama; Guido Yunior García Loor; Nancy Monserrate Macías Palacios; Paulo Gregorio Flores Tacle; Anthony James Chávez Salazar

\begin{abstract}
Some cardiovascular diseases (CVD) are usually acute phenomena caused by obstructions that make it impossible for blood to flow to the brain or heart, such is the case of heart attacks, as they cause a clot that blocks the coronary artery that carry blood and oxygen to the heart Following this blood blockage, lack of oxygen arises, so that the heart cells die and thus the damage to the heart muscle is generated, this whole process being known as ischemia. Depending on the degree of obstruction of the artery (total or partial) it will be the damage or consequence for the organism, that is, in those cases of severe obstruction of the coronary artery and suddenly, it could cause a heart attack or myocardial infarction. The interest to develop the present investigation that, having as main objective the exposition of the fundamental aspects associated to the appropriate procedures in the ICU to treat patients with ischemia and myocardial infarction, and based on this, it has been chosen to advance an investigation under a bibliographic design, with a review methodology. The conclusions highlighted the requirement that, today more than before, is demanded for the knowledge, prevention and treatments, not only of the critical patient, but especially of the possible complications associated with cardiovascular disease that by the way can become potentially serious, and cause death, however, some of these complications can be prevented in a simple way, as for example, with the management and constant updating of clinical practice guidelines developed based on evidence proven in the ICU. However, the diagnosis and early treatment of ischemia and myocardial infarction are the key to avoiding the irreversible consequences of the disease, as well as the follow-up of the appropriate procedures in the ICU represent the best alternative for the prevention of complications and recovery patient total.
\end{abstract}

Key Words: Cardiovascular; Obstructions; Artery; Cells; Patient. 


\section{Procedimientos adecuados para pacientes con isquemia e infarto miocardio al ingreso en la UCI}

Vol. 3, núm. 3., (2019)

Olga Mariana Villavicencio Chica; Flor María Buri Satama; Guido Yunior García Loor; Nancy Monserrate Macías Palacios; Paulo Gregorio Flores Tacle; Anthony James Chávez Salazar

\section{Introducción.}

La isquemia miocárdica es una enfermedad del corazón que se produce cuando el flujo de sangre que se dirige a este órgano, se reduce, causando deficiencia en el oxígeno recibido. Esta reducción, por lo general, se origina por causa de una obstrucción parcial o total en las arterias coronarias. (Mayo Clinic, 2018) (Bravo Vidal, Andrade Sánchez, Cedeño Arteaga, \& Castillo Silva, 2018)

Dependiendo del grado de obstrucción de la arteria será el daño o consecuencia para el organismo, por ejemplo, en aquellos casos de obstrucción grave de la arteria coronaria y de forma repentina, podría causar un ataque cardíaco o infarto al miocardio.

"Los ataques cardíacos son provocados por un coágulo que bloquea la arteria coronaria. Las arterias coronarias llevan sangre y oxígeno al corazón. Si el flujo sanguíneo se bloquea el corazón sufre por falta de oxígeno y las células cardíacas mueren”. (A.D.A.M. - Enciclopedia Médica, 2019)

Vitón, Lorenzo, Linares, Lazo, \& Godoy (2018) señala que el infarto agudo de miocardio (IMA) es uno de los dos formatos clínicos más frecuentes en que se deriva la cardiopatía isquémica (CI). Este autor afirma que este tipo de complicaciones provocaban, a principios del siglo $\mathrm{XX}$, menos del $10 \%$ de todas las muertes en el mundo, dato que contrasta con el presente, pues ahora representan casi el 50\% de los decesos en los países desarrollados, y el $25 \%$ en los países en vías de desarrollo. Indicó que la OMS, pronostica que la enfermedad isquémica del corazón será responsable de 11,1 millones de muertes para el 2020. (párr. 3-4)

Actualmente, la principal causa de fallecimiento a nivel mundial corresponde a las enfermedades 


\section{Procedimientos adecuados para pacientes con isquemia e infarto miocardio al ingreso en la UCI}

Vol. 3, núm. 3., (2019)

Olga Mariana Villavicencio Chica; Flor María Buri Satama; Guido Yunior García Loor; Nancy Monserrate Macías Palacios; Paulo Gregorio Flores Tacle; Anthony James Chávez Salazar

cardiovasculares (ECV), se estima que cada vez mueren más personas por patologías asociadas con la salud del corazón que por cualquier otra causa. (Fundación Española del Corazón, 2018)

En cuanto a las cifras, se estima que en el año 2015 murieron por causa de ECV un aproximado de 17,7 millones de personas, que representa un $31 \%$ del total de las muertes reportadas en todo el mundo. De estas defunciones, se estima que un total de 7,4 millones fueron por causa de cardiopatía coronaria, y un total de 6,7 millones, a los accidentes cardiovasculares (ACV). (Organización Mundial de la Salud, 2017)

Recientes datos aportados por la Organización Mundial de la Salud [OMS] (2019) refieren que "las enfermedades cardiovasculares (ECV) son un conjunto de trastornos del corazón y de los vasos sanguíneos" que representan la causa principal de defunción en todo el mundo, matando personas con una marcada diferencia (más del $80 \%$ ) en los países de bajos y medianos ingresos. "El consumo de tabaco, una dieta malsana y la inactividad física aumentan el riesgo de infartos de miocardio" (OMS, 2019)

Es fundamental la rapidez con que se diagnostique un infarto al miocardio, ya que de ello va a depender la vida y la reducción de complicaciones del paciente. Para Muñoz, Valladares, Francisco, \& González (2016) estos pacientes requieren una serie de cuidados y atenciones especiales tales como: "monitorización eléctrica continua, colocando un monitor desfibrilador cerca del paciente, acceso intravenoso periférico y monitorización no invasiva de la saturación de oxígeno". Asimismo, se maneja dentro de los principales tratamientos el alivio del dolor, oxigeno, entre otros. (p. 176) 


\section{Procedimientos adecuados para pacientes con isquemia e infarto miocardio al ingreso en la UCI}

Vol. 3, núm. 3., (2019)

Olga Mariana Villavicencio Chica; Flor María Buri Satama; Guido Yunior García Loor; Nancy Monserrate Macías Palacios; Paulo Gregorio Flores Tacle; Anthony James Chávez Salazar

En virtud de lo anterior, generalmente, estos pacientes deben ser ingresados con rapidez a una Unidad de Terapia Intensiva (UTI) o Unidad de Cuidados Intensivos (UCI) a los fines de monitorear constantemente la función cardíaca dado que las arritmias constituyen la principal causa de muerte tras las primeras horas posterior al ataque cardíaco. Asimismo, en esta área especializada se encarga de la terapéutica adecuada que deben recibir estos pacientes. Existen en algunos centros hospitalarios, Unidades de Cuidados Intensivos Coronarios, que son igualmente áreas especializadas, ideales para el cuidado y aplicación de los procedimientos adecuados que garanticen al paciente que presentan enfermedades graves del corazón, mayores posibilidades de evitar complicaciones y de recuperación. (Peña Moran, Decker Larrea, \& Pino Pin, 2019)

El tratamiento médico adecuado e inmediato, puede reducir o evitar el daño, no obstante, la tardanza en la atención especial y adecuada posibilita que alguna sección del músculo cardíaco muera y el daño al corazón sea irreversible.

Es de antes expuesto que nace el interés por desarrollar la presente investigación que, teniendo como objetivo principal la exposición de los aspectos fundamentales asociados a los procedimientos adecuados en UCI para tratar pacientes con isquemia e infarto al miocardio, tratará otras concepciones básicas tales como: qué es isquemia e infarto miocárdico, tipos, criterios diagnósticos, entre otros, considerados necesarios no solo para comprensión del tema, sino también para la ampliación de dichos conocimiento y para el fomento de posteriores investigaciones en cuanto a lo que implica el manejo de este tipo especial de pacientes tras su ingreso a la mencionada unidad. 


\section{Procedimientos adecuados para pacientes con isquemia e infarto miocardio al ingreso en la UCI}

Vol. 3, núm. 3., (2019)

Olga Mariana Villavicencio Chica; Flor María Buri Satama; Guido Yunior García Loor; Nancy Monserrate Macías Palacios; Paulo Gregorio Flores Tacle; Anthony James Chávez Salazar

\section{Materiales y Métodos.}

En la realización de este trabajo, ciertos computadores personales y la conexión a internet se han concebido como materiales fundamentales, recursos que sirvieron para efectuar la búsqueda pormenorizada de fuentes de información en diversas bases de datos sobre los procedimientos adecuados en pacientes con isquemia e infarto miocardio al ingresar en la unidad de cuidados intensivos (UCI). A tales fines se ha escogido adelantar una investigación bajo un diseño bibliográfico, con una metodología de revisión.

A partir del 15 de agosto de 2019, se realizó una búsqueda estructurada de literatura cientificoacadémica en las bases de datos PubMed, LILACS, Embase, SciELO, Dialnet, MedlinePlus, EBSCO, ScienceDirect, Redib, Cochrane Library y otras, con las expresiones "isquemia e infarto miocardio en UCI"; "Cuidados intensivos de la isquemia e infarto miocardio" y "Cuidados intensivos coronarios"; además de los términos "isquemia miocardia"; "infarto miocardio"; "unidad de cuidados intensivos" y otras locuciones técnicas propias del tema en cuestión, con las que se logró obtener un considerable números de resultados de búsqueda referente a títulos de diversa índole bibliográfica.

En función de determinar los referidos procedimientos adecuados, en otras palabras, el manejo en UCI de pacientes con esa condición especial, a dichos resultados se aplicó una serie de filtros, que gradualmente fue reduciendo el total de fuentes documentales resultantes en cada base de datos consultada.

Entre los múltiples filtros preestablecidos en cada interfaz de búsqueda de las diferentes bases de 


\section{Procedimientos adecuados para pacientes con isquemia e infarto miocardio al ingreso en la UCI}

Vol. 3, núm. 3., (2019)

Olga Mariana Villavicencio Chica; Flor María Buri Satama; Guido Yunior García Loor; Nancy Monserrate Macías Palacios; Paulo Gregorio Flores Tacle; Anthony James Chávez Salazar datos utilizadas, fundamentalmente se ejecutaron aquellos que arrojaban obras clasificadas como: artículos científicos originales y de revisión, guías de manejo, revisiones sistemáticas con o sin metaanálisis, estudios de cohorte, ensayos clínicos, protocolos, libros y libros electrónicos, boletines y folletos de reconocidas organizaciones internacionales y nacionales, tesis de posgrado y doctorado, noticias científicas y otros documentos e información con destacado valor cientificoacadémico.

Así mismo, se aplicaron los filtros que mostraran sólo las publicaciones hechas entre 2009 y 2019, haciendo la excepción con algunas fuentes por considerar que su contenido aún se encuentra vigente. Se omitieron fuentes que no estuvieron redactadas en castellano, salvo algunos casos de obras en inglés; así como también aquellos que no se refieren al género humano. Se desestimaron estudios de casos y controles; series y reportes de casos; editoriales, cartas al editor y otros tipos de publicaciones de poca relevancia y escaso valor científico académico.

En definitiva, todo este proceso facilitó la escogencia de solamente aquellos trabajos de contenido reciente y relevantes por su correlación temática. A partir de allí, el equipo procede a darle la correspondiente lectura crítica y análisis de toda la evidencia seleccionada, lo que resultó consecutiva y consensuadamente en el fundamento de las ideas y planteamientos aquí plasmados.

\section{Resultados.}




\section{Procedimientos adecuados para pacientes con isquemia e infarto miocardio al ingreso en la UCI}

Vol. 3, núm. 3., (2019)

Olga Mariana Villavicencio Chica; Flor María Buri Satama; Guido Yunior García Loor; Nancy Monserrate Macías Palacios; Paulo Gregorio Flores Tacle; Anthony James Chávez Salazar

Según Delgado et al. (2019) algunas enfermedades cardiovasculares como es el caso de los ataques al corazón, suelen ser fenómenos agudos originados por obstrucciones que imposibilitan que la sangre fluya hacia el cerebro o el corazón. (p. 686)

La isquemia miocárdica, también llamada isquemia cardíaca, se origina cuando baja el flujo de sangre que va al corazón, lo que a su vez embarga la suficiente recepción de oxígeno. Generalmente, la referida reducción del flujo se produce por la obstrucción parcial o total de las arterias del corazón (arterias coronarias). Consecuentemente esta condición puede causar daño al músculo cardíaco y oprimir su capacidad de bombear sangre de forma eficaz, así como también, provocar ritmos cardíacos anormales graves. Cuando la obstrucción de una arteria coronaria llega a ser grave y repentina, puede producir un ataque cardíaco. (Mayo Clinic, 2018)

Por su parte, Moreno \& del Portillo (2016), en el mismo orden de ideas, dicen que la isquemia miocárdica depende de diversos factores moleculares y fisiológicos, para que pueda considerarsele como reversible o irreversible.

En la reversible puede deberse a isquemia de demanda, que es la ocasión en la que las células del miocardio incrementa su demanda de oxígeno, o a isquemia de suplencia, referida a la obstrucción de uno de los vasos epicárdicos. Cualquier aumento en los requerimientos de oxígeno del miocardio debería equilibrarse con una adición en el flujo.

La extracción del oxígeno por las células es máximo en las condiciones de reposo basal. El no balance en la demanda y la suplencia desencadena la isquemia. Si se mantiene, la isquemia juega una serie de cambios bioquímicos dados por cambios físicos del miocardio, 


\section{Procedimientos adecuados para pacientes con isquemia e infarto miocardio al ingreso en la UCI}

Vol. 3, núm. 3., (2019)

Olga Mariana Villavicencio Chica; Flor María Buri Satama; Guido Yunior García Loor; Nancy Monserrate Macías Palacios; Paulo Gregorio Flores Tacle; Anthony James Chávez Salazar conocido esto como la cascada isquémica.[...] Conceptos como el miocardio aturdido (disfunción mecánica temporal luego de una lesión isquémica pero con flujo sanguíneo normal en ausencia de cualquier lesión irreversible) y el miocardio hibernante (región miocárdica viable, sin contractilidad) son formas quiescentes de la función cardiaca y explican un poco la capacidad del miocardio de restablecer su funcionamiento normal luego de un episodio de isquemia.(p.500,502)

Los mismos explican que, en la isquemia miocárdica irreversible se presentan tres tipos de muerte celular a nivel miocárdico: la necrosis, la apoptosis y la autofagia, tradicionalmente controladores de "la muerte celular y la perduración de las vías que generan estos procesos, que son distintas y diferentes". La necrosis, mayormente mediadora en la muerte irreversible, "es una muerte celular no controlada, accidental y regulada eficientemente por la pérdida celular intrínseca para mantener o guardar la viabilidad celular". Por otra parte, la muerte celular programada bien controlada, por mecanismos moleculares tanto extrínsecos como intrínsecos, es lo que se entiende como apoptosis. La autofagia, considerado un reciente y muy atractivo concepto para referir la muerte celular, "es un mecanismo de sobrevida celular, inicialmente mediado por la degradación y reciclaje de organelas celulares que cuando fracasa lleva a un exceso en la degradación celular y desencadena un mecanismo de muerte celular". (Moreno \& del Portillo; 2016; p. 501)

En otro orden de ideas, de Pérez (2019) se comprende que, es impreciso que frecuentemente se asuma que el infarto de miocardio (IM/IAM) es sinónimo de infarto, ya que en realidad, éste último se refiere a una concepción más general, pues se trata de la muerte celular (necrosis) de todo o parte de un órgano cualquiera por falta de irrigación sanguínea, debiéndose tal insuficiencia 


\section{Procedimientos adecuados para pacientes con isquemia e infarto miocardio al ingreso en la UCI}

Vol. 3, núm. 3., (2019)

Olga Mariana Villavicencio Chica; Flor María Buri Satama; Guido Yunior García Loor; Nancy Monserrate Macías Palacios; Paulo Gregorio Flores Tacle; Anthony James Chávez Salazar

de sangre a una obstrucción total o parcial (aterosclerosis: endurecimiento o estenosis: estrechez) de la arteria correspondiente; mientras que el IM igualmente se debe a un insuficiente riego sanguíneo debido a la obstrucción de una arteria pero específicamente del músculo cardiaco, he allí su particularidad.

Desde otra perspectiva, la Sociedad Española de Medicina Interna [SEMI] (2019) expone que el infarto consiste en la obstrucción brusca del paso de sangre a lo largo de una arteria coronaria con la subsiguiente muerte de la parte del corazón que es irrigada (alimentada) por dicha arteria, y por ello esta condición es particularmente considerada de urgencia, asociada a una elevada mortalidad. Continúan detallando que se debe considerar que existen dos tipos de infarto de miocardio, que son: con onda $Q$ (o síndrome coronario agudo con elevación del segmento ST), que se produce por la obstrucción prolongada de una de las arterias coronarias importantes, lo que lleva a la muerte de una zona más o menos grande del corazón; y, sin onda $Q$, en el que la falta de irrigación generalmente afecta a arterias del corazón un poco más pequeñas, haciendo posible que no muera una zona tan extensa del corazón y a su vez favorezca un mejor pronóstico. El infarto de miocardio sin onda Q se agrupa junto a la angina inestable, dentro de los síndromes coronarios agudos sin elevación del segmento ST. Respecto a ambas definiciones, cabe aclarar que la "onda Q" está referida a una pequeña cicatriz que puede observarse en el electrocardiograma una vez que ha pasado la fase aguda del infarto. (SEMI, 2019)

El Hospital Universitario Austral (2019) refiere que esta condición, que también es comúnmente conocida como ataque cardíaco, se produce por una exigua irrigación sanguínea al corazón, y por ende, falta de oxígeno. Ésa situación es llamada: isquemia, que puede originarse por varias razones, 


\section{Procedimientos adecuados para pacientes con isquemia e infarto miocardio al ingreso en la UCI}

Vol. 3, núm. 3., (2019)

Olga Mariana Villavicencio Chica; Flor María Buri Satama; Guido Yunior García Loor; Nancy Monserrate Macías Palacios; Paulo Gregorio Flores Tacle; Anthony James Chávez Salazar

entre las que destacan: a) engrosamiento de las paredes de las arterias coronarias, debido una progresiva acumulación de grasa (placa), que a su vez es a causa del colesterol, y que es posible que pueda llegar al punto taparse; b) por un coágulo, ocurrido en otra parte del organismo, que bloquea una arteria coronaria (a veces por el engrosamiento de placa), que impide llevar sangre al corazón; c) sangramiento y formación coágulo en el interior de la arteria, que ocurre a raíz del rompimiento de la placa; y d) por espasmo (de los que aún se desconocen sus causas) en una arteria coronaria, entendido como una contracción que dificulta o interrumpe el flujo sanguíneo. Evidentemente, las células cardíacas mueren si el flujo sanguíneo se bloquea y el órgano no recibe sangre ni oxígeno.

Bazzino (2013) expone que en el año 2000 nació el documento que redefinió el concepto infarto de miocardio (IM) y mediante el mismo se logró un consenso general de que dicho término se usaría en base a la consideración de atributos tales como:

[...] el monto de la pérdida de miocitos (tamaño del infarto), las circunstancias en las que se presenta (espontáneo o en el contexto de una intervención coronaria) y el tiempo de evolución de la necrosis en relación con el momento de la observación. (p. 403)

Continua el tratadista exponiendo que, luego, en 2007, se destacan otros dos aspectos fundamentales para presentar una nueva y universal definición de IM, que por una parte enfatizaba la importancia de la troponina en el diagnóstico, y, por la otra, definía cinco tipos de infarto (que más adelante se mencionarán). Posteriormente, en 2012, debido a la necesidad de actualizar la anterior definición universal por las limitaciones propias que se evidenciaban en su conceptualización, y al desarrollo de herramientas diagnósticas y el mejor entendimiento del valor, 


\section{Procedimientos adecuados para pacientes con isquemia e infarto miocardio al ingreso en la UCI}

Vol. 3, núm. 3., (2019)

Olga Mariana Villavicencio Chica; Flor María Buri Satama; Guido Yunior García Loor; Nancy

Monserrate Macías Palacios; Paulo Gregorio Flores Tacle; Anthony James Chávez Salazar

fue que surgió la tercera definición universal de IM en la que se destacan como aspectos centrales:

“[...] los síntomas clínicos, los biomarcadores cardíacos y las alteraciones electrocardiográficas compatibles con isquemia miocárdica". (p. 403-404)

Por lo tanto, alega el experto, es así como con la tercera definición universal se hace énfasis en:

- El importante significado que tiene la elaboración de la historia clínica de síntomas isquémicos en un lapso de no menos de 20 minutos, no necesariamente fundados en el típico dolor torácico.

- El análisis de los escenarios clínicos en los que ocurre el IM.

- El papel preponderante que, primeramente, siguen representando los biomarcadores en el diagnóstico, y segundo, la mejor ponderación que le es reconocida a las pruebas con imágenes.

Tipos de IM/IAM.

Calvachi et al. (2017) cita a (Jneid, Alam, Virani \& Bozkur; 2013) para indicar que la Organización Mundial de la Salud, con fines diagnósticos, epidemiológicos e investigativos, desde 1971 ha procurado definir mejor esta entidad. Continúa diciendo, en base a (White, Thygesen, Alpert \& Jaffe; 2014), que ha sido en razón de lo antes indicado que, a lo largo de este tiempo, se han originado diversas clasificaciones, y una de esas es la tercera definición de infarto, no obstante, en una previa investigación, Thygesen et al. (2013) clasificaron el IAM de la siguiente manera: 


\section{Procedimientos adecuados para pacientes con isquemia e infarto miocardio al ingreso en la UCI}

Vol. 3, núm. 3., (2019)

Olga Mariana Villavicencio Chica; Flor María Buri Satama; Guido Yunior García Loor; Nancy Monserrate Macías Palacios; Paulo Gregorio Flores Tacle; Anthony James Chávez Salazar

- Tipo 1: infarto agudo de miocardio espontáneo.

- Tipo 2: infarto agudo de miocardio secundario a desequilibrio isquémico.

- Tipo 3: infarto agudo de miocardio que conduce a muerte cuando aún no se dispone de los resultados de biomarcadores.

- Tipo 4 A: infarto agudo de miocardio relacionado con intervención coronaria percutánea.

- Tipo 4 B: infarto agudo de miocardio relacionado con trombosis del stent.

- Tipo 5: infarto agudo de miocardio relacionado con derivación aorto-coronaria con injerto.

- (Calvachi et al.; 2017; p. 593)

Criterios generales para el diagóstico de IAM.

Bazzino (2013) indica que "[...] el término infarto agudo de miocardio (IAM) debe ser utilizado cuando existe evidencia de necrosis miocárdica en un escenario clínico consistente con isquemia miocárdica aguda". Es por ello que, continua el experto, en base a tales condiciones que cualquier criterio que se menciona a continuación aplicarán para un diagnóstico de IAM:

$1^{\circ}$. Detección de un incremento y/o caída de los niveles de biomarcadores cardíacos (preferentemente troponinas), con al menos un valor por encima del percentil 99 del límite superior de referencia, $\mathrm{y}$, al menos, una de las siguientes condiciones:

- Síntomas de isquemia. 


\section{Procedimientos adecuados para pacientes con isquemia e infarto miocardio al ingreso en la UCI}

Vol. 3, núm. 3., (2019)

Olga Mariana Villavicencio Chica; Flor María Buri Satama; Guido Yunior García Loor; Nancy Monserrate Macías Palacios; Paulo Gregorio Flores Tacle; Anthony James Chávez Salazar

- Cambios nuevos, o presumiblemente nuevos, en el segmento ST y/o onda T; o bloqueo de rama izquierda nuevo.

- Desarrollo de ondas Q patológicas.

- Evidencia imagenológica de una pérdida nueva de miocardio viable, o de una anormalidad nueva en la contractilidad parietal regional.

- Identificación de trombo intracoronario mediante angiografía o autopsia.

$2^{\circ}$. Muerte cardíaca con síntomas sugestivos de isquemia miocárdica y alteraciones electrocardiográficas isquémicas o bloqueo de rama izquierda nuevo. La muerte ocurre antes de la dosificación de biomarcadores o antes que los valores de estos alcancen niveles anormales.

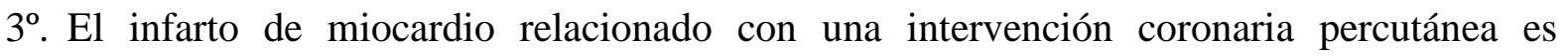
arbitrariamente definido como una elevación de troponinas cinco veces el valor del percentil 99 del nivel de referencia, o > 20\% si los niveles basales están elevados (estables o en descenso). Adicionalmente se requiere alguna de las siguientes condiciones: a) síntomas sugestivos de isquemia miocárdica; b) nuevos cambios electrocardiográficos isquémicos; c) hallazgos angiográficos consistentes con una complicación del procedimiento, o d) imágenes que evidencien una pérdida nueva de miocardio viable o alteraciones sectoriales nuevas de la contractilidad.

$4^{\text {o }}$. Trombosis del stent relacionado a infarto de miocardio, detectado por angiografía o 


\section{Procedimientos adecuados para pacientes con isquemia e infarto miocardio al ingreso en la UCI}

Vol. 3, núm. 3., (2019)

Olga Mariana Villavicencio Chica; Flor María Buri Satama; Guido Yunior García Loor; Nancy Monserrate Macías Palacios; Paulo Gregorio Flores Tacle; Anthony James Chávez Salazar autopsia en el con texto de isquemia miocárdica, y con un ascenso y/o descenso de los niveles de biomarcadores cardíacos, con al menos un valor por encima del percentil 99 del rango de referencia.

$5^{\circ}$. El infarto de miocardio relacionado con cirugía de revascularización miocárdica es arbitrariamente definido como una elevación superior a diez veces el percentil 99 del rango de referencia en pacientes con niveles basales normales, y con al menos una de las siguientes condiciones: a) ondas Q patológicas o bloqueo de rama izquierda nuevo; b) nueva oclusión de puente o arteria nativa documentada angiográficamente, o c) imágenes que evidencien una pérdida nueva de miocardio viable o alteraciones sectoriales nuevas de la contractilidad. (Bazzino; 2013; p. 405)

\section{Cuidados asistenciales en UCI.}

El paciente crítico es definido por la Sociedad Americana de Medicina Intensiva, como aquel que se encuentra fisiológicamente inestable, que requiere soporte vital avanzado y una evaluación clínica estrecha con ajustes continuos de terapia según evolución. La unidad de cuidados críticos (UCI) es sin duda el lugar que está dotado del personal humano y la infraestructura adecuada para cumplir con los requerimientos de cuidado anteriormente descritos. [...] Los cuidados que deben tener los pacientes ingresados a las unidades de cuidados intensivos (UCI), son muy importantes, para evitar complicaciones que comprometan aún más la vida de los pacientes. En los entornos de clínicas y hospitales en donde existen unidades de cuidados intensivos hay que tener muchas previsiones sanitarias para evitar la proliferación de bacterias e infecciones y a su vez, cuidar el metabolismo de los pacientes para que no haya descompensaciones. (Briones et al.; 2019) 


\section{Procedimientos adecuados para pacientes con isquemia e infarto miocardio al ingreso en la UCI}

Vol. 3, núm. 3., (2019)

Olga Mariana Villavicencio Chica; Flor María Buri Satama; Guido Yunior García Loor; Nancy

Monserrate Macías Palacios; Paulo Gregorio Flores Tacle; Anthony James Chávez Salazar

Tratamiento o procedimientos a seguir en pacientes ingresados a la UCI por infarto al miocardio

Medidas Generales y Complementarias.

Es importante mencionar previamente, acerca de las Guías de Práctica Clínica (GPC) que son instrumentos fundamentales en las diferentes áreas hospitalarias las cuales permiten, con base a la evidencia relevante sobre un tema particular, ayudar a los médicos tratantes para determinar la mejor estrategia en la medida de los posible para tratar a sus pacientes. Estas guías se elaboran tomando en consideración no sólo el resultado final del tratamiento, sino que debe sopesar los factores de riesgos contra los beneficios que presenta un procedimiento diagnóstico o terapéutico en particular.

Coll, Valladares, \& González (2016) actualizaron una guía práctica para tratar el infarto agudo de miocardio, en Cuba, donde basándose en recomendaciones y niveles de evidencia, de forma similar a las diferentes GPC internacionales, mencionan en cuanto al manejo y tratamiento intrahospitalario del paciente en la Unidad de Cuidados Intensivos Coronarios, específicamente de las medidas generales y complementarias:

Monitorización electrocardiográfica continua hasta 48-72 horas (más allá si complicaciones). Oximetría de pulso: en las primeras 6 horas (más allá si congestión pulmonar o inestabilidad hemodinámica). Signos vitales cada 1 hora (primeras 6 horas); cada 2 horas (resto de las primeras 24 horas) y luego cada 3-4 horas, en casos no complicados. En la sección de camas convencionales se indicarán por cada turno. Medir diuresis cada 3 horas en las primeras 24 horas y luego cada turno en casos no complicados. 


\section{Procedimientos adecuados para pacientes con isquemia e infarto miocardio al ingreso en la UCI}

Vol. 3, núm. 3., (2019)

Olga Mariana Villavicencio Chica; Flor María Buri Satama; Guido Yunior García Loor; Nancy Monserrate Macías Palacios; Paulo Gregorio Flores Tacle; Anthony James Chávez Salazar

En cuanto a las complementarias: Dentro de las principales se mencionan las medidas generales de rutina al ingreso (hemograma, glucemia, ionograma, coagulograma, perfil lípídicos que se repetirán solo en base a las características del paciente o complicaciones). Rayos x de tórax (al ingreso y repetir si aparecieran complicaciones o después de cateterismo venoso profundo) ECG (a los 90 minutos del inicio del tratamiento trombolítico y luego c/24 horas, si existiera frecuencia mayor o si hubiera complicaciones). Biomarcadores séricos (CPK total y CK-MB) c/8 horas en las primeras 24 horas, después diario si es necesario hasta las 48,72 horas. Ecocardiografía (precoz para la toma de decisiones si ECG no es diagnóstico, de inmediato si existe sospecha de complicación mecánica, y de rutina en algún momento de la hospitalización, y ante la sospecha de enfermedad cardiaca asociada. Alivio máximo del dolor Morfina: dosis de 4-8 mg IV y posteriormente repetir dosis de 2-8 $\mathrm{mg}$ a intervalos de 5-15 min hasta que desaparezca el dolor o se observe una toxicidad clara. (p. 178)

\section{Tratamiento farmacológico.}

La Sociedad Española de Cardiología (2017) mediante una guía estableció un proceso para el manejo del Síndrome Coronario Agudo sin Elevación del ST (SCASEST) Estándar de Calidad SEC en el cual refieren que es recomendable "mantener al paciente en un ambiente tranquilo en reposo y la administración de oxígeno en el caso de saturación menor del 90\% o la presencia de signos de insuficiencia cardiaca". En cuanto a la farmacología indican que para el alivio del dolor que persiste a pesar de medicación antianginosa serían la administracion de opiáceos tales como 


\section{Procedimientos adecuados para pacientes con isquemia e infarto miocardio al ingreso en la UCI}

Vol. 3, núm. 3., (2019)

Olga Mariana Villavicencio Chica; Flor María Buri Satama; Guido Yunior García Loor; Nancy Monserrate Macías Palacios; Paulo Gregorio Flores Tacle; Anthony James Chávez Salazar

la morfina. El tratamiento antianginoso debe combinar nitratos y betabloqueantes. Es importante la monitorización adecuada de la presión arterial mientras se administra y debe considerarse no administrar si el paciente ha consumido sildenafilo en las últimas $24 \mathrm{~h}$ o taldalafilo en las $48 \mathrm{~h}$ previas dado el riesgo de hipotensión severa. Este tratamiento a base de betabloqueante por una parte proporciona control sintomático el cual se ha asociado a reducción de la mortalidad intrahospitalaria. No se debe administrar betabloqueantes en aquellos pacientes en los que se sospeche de consumo reciente de cocaína o se sospeche de vasoespasmo coronario. Asimismo, se recomienda en los pacientes con diagnóstico de SCASEST iniciar el tratamiento con estatinas de alta potencia a dosis altas, excepto contraindicación. (p. 33,34)

Manejo de pacientes con Isquemia e Infarto de miocardio en UCI.

De conformidad a la obra de Borrayo et al. (2017), que trata sobre la adaptación del protocolo conocido en otros países como "Código infarto" a la medicina institucional mexicana; útil para la organización los "servicios de urgencias y admisión continua", la asignación de "las actividades específicas al personal que participa en la atención del paciente" y la garantía del "diagnóstico y tratamiento al paciente que demanda atención de urgencias por infarto agudo de miocardio"; se logran conocer las responsabilidades, que según los tratadistas, son propias del especialista de la Unidad de Cuidados Intensivos (UCI), jefe(a) de servicio o responsable del paciente en cuestión, siendo éstas las siguientes:

- Organiza el servicio de manera que se garantice la atención a pacientes con código infarto, asigna cama(s) de uso preferente. 


\section{Procedimientos adecuados para pacientes con isquemia e infarto miocardio al ingreso en la UCI}

Vol. 3, núm. 3., (2019)

Olga Mariana Villavicencio Chica; Flor María Buri Satama; Guido Yunior García Loor; Nancy

Monserrate Macías Palacios; Paulo Gregorio Flores Tacle; Anthony James Chávez Salazar

- Recibe al paciente.

- Establece el manejo para el código infarto.

- Monitorea la evolución y estratifica tempranamente al paciente.

- Supervisa que se continúe completo el llenado del RENASCA.

- Gestiona que se envíe a piso de hospitalización o a segundo nivel de atención a los pacientes con Código infarto tratados exitosamente en las siguientes 48 a 72 horas.

- De estar disponible en la unidad, inicia la rehabilitación cardiaca temprana desde las primeras 24 o 48 horas.

- (Borrayo et al.; 2017; p. 237)

\section{Conclusión.}

Hoy en día, el manejo clínico de los pacientes ingresados en UCI como consecuencia de cardiopatías críticas, entre ellas la isquemia y el infarto al miocardio, exige cada día actualidad en conocimientos, prevención y tratamientos de posibles complicaciones asociadas con la enfermedad cardiovascular y la condición general del paciente crítico, las cuales pueden llegar a ser potencialmente graves, pudiendo causar incluso la muerte, no obstante, algunas de estas complicaciones pueden prevenirse de manera sencilla.

La actualización y manejo puntual de las guías prácticas clínicas elaboradas con base a evidencias comprobadas colaboran con el personal médico de la UCI en la toma de decisiones y representan 


\section{Procedimientos adecuados para pacientes con isquemia e infarto miocardio al ingreso en la UCI}

Vol. 3, núm. 3., (2019)

Olga Mariana Villavicencio Chica; Flor María Buri Satama; Guido Yunior García Loor; Nancy Monserrate Macías Palacios; Paulo Gregorio Flores Tacle; Anthony James Chávez Salazar

una mayor oportunidad de supervivencia y recuperación del paciente cardiópata.

El diagnóstico y tratamiento precoz de las enfermedades cardiovasculares graves (isquemia e infarto al miocardio) son la clave para evitar las consecuencias irreversibles de la enfermedad, así como el seguimiento de los procedimientos adecuados en la UCI representan la mejor alternativa para la prevención de complicaciones y la recuperación total del paciente.

\section{Bibliografía.}

A.D.A.M. - Enciclopedia Médica. (31 de Julio de 2019). MedlinePlus. Recuperado el 17 de 08 de 2019, de medlineplus.gov: https://medlineplus.gov/spanish/ency/article/000195.htm

Bazzino, Ó. (Diciembre de 2013). Tercera definición universal de infarto de miocardio. Revista Uruguaya de Cardiología, 28(3), 403 - 411. Recuperado el 23 de 07 de 2019, de http://www.scielo.edu.uy/scielo.php?pid=S1688-

04202013000300014\&script=sci_arttext\&tlng=en\#1

Borrayo, G., Pérez, G., Martínez, O., Almeid, E., Ramírez, E., Estrada, J., . . Arriaga, J. (2017). Protocolo para atención de infarto agudo de miocardio en urgencias: Código infarto. Rev Med Inst Mex Seguro Soc, 55(2), 233-246. Recuperado el 15 de 08 de 2019, de https://www.medigraphic.com/pdfs/imss/im-2017/im172q.pdf

Bravo Vidal, A., Andrade Sánchez, T., Cedeño Arteaga, E., \& Castillo Silva, M. (2018). Cardiopatía Isquémica, Enfermedad Prevenible. RECIMUNDO, 2(3), 550-563.

Briones, Á., Holguín, L., Vallejo, P., Santana, O., Jiménez, M., \& Villavicencio, G. (2019). Cuidados asistenciales en pacientes ingresados en UCI. Reciamuc, 3(3), 465 - 486. doi:10.26820/reciamuc/3.(3).junio.2019.465-486

Calvachi, P., Barrios, D., Puccini, M., Mojica, H., Delgadillo, D., Gómez, M., . . Buitrago, A. (Noviembre - Deciembre de 2017). Frecuencia de los tipos de infarto agudo de miocardio según la tercera definición. Revista Colombiana de Cardiología, 24(6), 592-597. doi:10.1016/j.rccar.2017.06.008

Coll, Y., Valladares, F., \& González, C. (Junio de 2016). Infarto agudo de miocardio. Actualización de la Guía de Práctica. Finlay, 6(2), 170-190. Recuperado el 29 de Agosto de 2019, de http://scielo.sld.cu/pdf/rf/v6n2/rf10206.pdf 


\section{Procedimientos adecuados para pacientes con isquemia e infarto miocardio al ingreso en la UCI}

Vol. 3, núm. 3., (2019)

Olga Mariana Villavicencio Chica; Flor María Buri Satama; Guido Yunior García Loor; Nancy Monserrate Macías Palacios; Paulo Gregorio Flores Tacle; Anthony James Chávez Salazar

Delgado, J., Lara, V., Flores, L., Sabando, B., Aguilar, E., \& Fernández, G. (Abril de 2019). Patologías Específicas de Importancia en la U.C.I. RECIAMUC, 3(2), 665-687. $\begin{array}{llllll}\text { Recuperado el } 29 \text { de } & \text { Agosto de }\end{array}$ http://reciamuc.com/index.php/RECIAMUC/article/view/360/374

Fundación Española del Corazón. (28 de Septiembre de 2018). fundaciondelcorazon.com. Recuperado el 29 de Agosto de 2019, de https://fundaciondelcorazon.com/blog-impulsovital/3264-las-cifras-de-la-enfermedad-cardiovascular.html

Hospital Universitario Austral. (2019). Infarto Agudo de Miocardio. Recuperado el 20 de 07 de 2019, de https://www.hospitalaustral.edu.ar/enfermedades/infarto-agudo-de-miocardio/

Mayo Clinic. (09 de Marzo de 2018). Mayo Clinic. Recuperado el 16 de 08 de 2019, de https://www.mayoclinic.org: https://www.mayoclinic.org/es-es/diseasesconditions/myocardial-ischemia/symptoms-causes/syc-20375417

Mayo Clinic. (09 de Marzo de 2018). mayoclinic.org. Recuperado el 20 de Agosto de 2019, de https://www.mayoclinic.org/es-es/diseases-conditions/myocardial-ischemia/symptomscauses/syc-20375417

Moreno, P., \& del Portillo, J. (Noviembre-Diciembre de 2016). Isquemia miocárdica: conceptos básicos, diagnóstico e implicaciones clínicas. Segunda parte. Revista Colombiana de Cardiología, 23(6), 500-506. doi:10.1016/J.RCCAR.2016.01.024

Muñoz, Y., Valladares, Francisco, \& González, C. (Junio de 2016). Infarto agudo de miocardio. Actualización de la Guía de Práctica. Finlay, 6(2), 170-190. Recuperado el 29 de Agosto de 2019, de http://scielo.sld.cu/pdf/rf/v6n2/rf10206.pdf

OMS. (2019). Enfermedades cardiovasculares. Recuperado el 24 de 07 de 2019, de Organización Mundial de la Salud: https://www.who.int/cardiovascular_diseases/es/

Organización Mundial de la Salud. (17 de Mayo de 2017). who.int/es. Recuperado el 29 de Agosto de 2019, de https://www.who.int/es/news-room/fact-sheets/detail/cardiovascular-diseases(cvds)

Peña Moran, J., Decker Larrea, P., \& Pino Pin, M. (2019). Utilidad del balón de contrapulsación en infarto agudo de miocardio. RECIMUNDO, 3(1), 850-867.

Pérez, P. (2019). Infarto de Miocardio. Recuperado el 22 de 07 de 2019, de https://fundaciondelcorazon.com/informacion-para-pacientes/enfermedadescardiovasculares/infarto.html

SEMI. (05 de Agosto de 2019). Infarto Agudo de Miocardio. Recuperado el 10 de 07 de 2019, de https://www.fesemi.org/informacion-pacientes/conozca-mejor-su-enfermedad/infartoagudo-de-miocardio 
Procedimientos adecuados para pacientes con isquemia e infarto miocardio al ingreso en la UCI

Vol. 3, núm. 3., (2019)

Olga Mariana Villavicencio Chica; Flor María Buri Satama; Guido Yunior García Loor; Nancy Monserrate Macías Palacios; Paulo Gregorio Flores Tacle; Anthony James Chávez Salazar

Sociedad Española de Cardiología. (2017). Síndrome Coronario Agudo sin Elevación del ST (SCASEST) Estándar de Calidad SEC Proceso. Guía de Práctica Clínica, España. Recuperado el 29 de Agosto de 2019, de https://secardiologia.es/images/SECExcelente/SCASEST_Proceso_20170104_Final.pdf

Vitón, A., Lorenzo, B., Linares, L., Lazo, L., \& Godoy, O. (2018). Caracterización clínicoepidemiológica de pacientes con infarto agudo del miocardio. Revista de Ciencias Médicas de Pinar del Río, 22(5), 24-33. Recuperado el 23 de 04 de 2019, de http://scielo.sld.cu/scielo.php?script=sci_arttext\&pid=S1561$31942018000500007 \& \operatorname{lng}=$ pt\&tlng=pt.

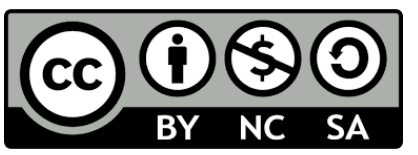

RECONOCIMIENTO-NOCOMERCIAL-COMPARTIRIGUAL

CC BY-NC-SA

ESTA LICENCIA PERMITE A OTROS ENTREMEZCLAR, AJUSTAR Y CONSTRUIR A PARTIR DE SU OBRA CON FINES NO COMERCIALES, SIEMPRE Y CUANDO LE RECONOZCAN LA AUTORÍA Y SUS NUEVAS CREACIONES ESTÉN BAJO UNA LICENCIA CON LOS MISMOS TÉRMINOS. 